\title{
A Contactless DC Tachogenerator with the Doubled Number of Phase Voltages
}

GRIDIN Vladimir M. (Moscow State Technical University named N.E. Bauman, Moscow, Russia) Associate Professor, Cand. Sci. (Eng.)

The article considers a tachogenerator containing a synchronous generator with a three-phase armature winding, a resistive doubler of the number of phase voltages, two bridge rectifiers of two three-phase voltages, and a resistive adder of the output voltages from two rectifiers. The requirements for the doubler and adder are defined. Expressions for the additional rectified three-phase voltage and for its increasing factor are obtained. Expressions for determining the sum of two rectified three-phase voltages and for the limit values of this sum are given. It has been found that the ripple of output voltage produced by the considered tachogenerator does not exceed 1,5\%, which is significantly lower than in the known tachogenerators $(3,5-4) \%$.

$\mathrm{K}$ e y w o rds: tachogenerator, phase voltage doubler, rectifiers, adder of rectifier voltages, output voltage ripple

\section{REFERENCES}

1. Osin I.L., Yuferov F.M. Elektricheskiye mashiny avtomaticheskikh ustroistv (Electrical machines of automatic devices). Moscow, Publ. of Moscow Power Engineering Institute, 2003, 424 p.

2. Putnikov V.V., Rybakov V.S., Slesarev A.V. Reversivnye beskontaktnye takhogeneratory postoyannogo toka (Reversible contactless DC tachogenerators). Moscow, Publ. «Informelektro», 1970, $32 \mathrm{p}$.

3. Brodovskii V.N., Karzhavov B.N., Rybkin Yu.P. Beskontaktnye takhogeneratory postoyannogo toka (Contactless DC tachogenerators). Moscow, Energoatomizdat, 1982, 128 p.
4. Tun A.Ya. Sistemy kontrolya skorosti elektroprivoda (Electric speed control systems). Moscow, Energoatomizdat, 1984, 169 p.

5. Gridin V.M. Elektrichestvo - in Russ. (Electricity), 2013, No. 6, pp. 42-46.

6. Gridin V.M. Elektrichestvo - in Russ. (Electricity), 2014, No. 5, pp. 34-38.

7. Gridin V.M. Elektrichestvo - in Russ. (Electricity), 2016, No. 4, pp. 56-61.

\section{Применение принципа обратной связи для решения уравнений трансформаторов и электрических машин}

\begin{abstract}
ЖУЛОВЯН в.В.
Предлагается учитывать активное сопротивление первичной обмотки, используя известный принцип обратной связи. При этом поиск состоит из двух этапов. На первом вводится напряжение за активным сопротивлением и, если в качестве независимых переменных вместо токов принять потокосцепления контуров, то порядок решаемой системы уравнений уменьшается на единииу, что во многих практических случаях позволяет существенно упростить и поиск, и вид искомых решений. На втором этапе, используя прием, известный как принцип обратной связи, находится математически строгое соотношение между условным и действительным напряжениями на зажимах. Таким образом можно представить предварительно найденные величины как функции последнего. Приводятся примеры, показывающие работоспособность и эффективность такого подхода на ряде задач, связанных с изучением установившихся и переходных режимов. электрических машин и трансформаторов.
\end{abstract}

К л ю ч е в ы е с л о в а: электрические машины, трансформаторы, первичная обмотка, активное сопротивление, обратная связь, порядок системы уравнений

Обратная связь - необходимый атрибут автоматической системы, она обеспечивает минимальное расхождение между задаваемой и воспроизводимой величинами [1]. Такой же прием в тех же целях может быть применен и при решении системы уравнений, описывающих некоторый объект. Рассмот- 
рим такую возможность при поиске строгого решения системы уравнений для трансформаторов и электрических машин, которые подобны при постоянной скорости вращения ротора [2].

Как известно, при их анализе в целях упрощения поиска искомого решения часто пренебрегают активным сопротивлением первичной обмотки $R_{1}$. Это происходит по двум причинам. Во-первых, влияние $R_{1}$ на электромеханическое преобразование энергии часто является косвенным, например через снижение напряжения, а во-вторых, малостью последнего, что обусловлено стремлением достичь высокого КПД. Возникающую ошибку обычно устраняют за счет увеличения сопротивления первичной обмотки, не затрагивая остальных величин [2]. Вместе с тем, погрешность от влияния $R_{1}$ легко устранить за счет приема, действующего по принципу обратной связи, получая при этом строгое решение.

Рассмотрим такую возможность на примере асинхронной машины с короткозамкнутым ротором, работающей от сети с уравновешенной системой напряжений при некоторой постоянной скорости $\omega$. Полагая, что сеть бесконечной мощности, уравнения машины в неподвижной системе координат имеют вид [3]:

$$
\begin{aligned}
& \mathbf{u}_{s}=\mathbf{i}_{s} R_{s}+p \Psi_{s} ; \\
& 0=\mathbf{i}_{r} R_{r}+p \Psi_{r}-j \omega \Psi_{r} ; \\
& \Psi_{s}=\mathbf{i}_{s} L_{s}+\mathbf{i}_{r} L_{m} ; \\
& \Psi_{r}=\mathbf{i}_{s} L_{m}+\mathbf{i}_{r} L_{r} .
\end{aligned}
$$

Здесь $p=d / d t ; \mathbf{u}_{s}, \mathbf{u}_{r}, \mathbf{i}_{s}, \mathbf{i}_{r}, \Psi_{s}, \Psi_{r}$ - векторы напряжений, токов и потокосцеплений статора (индекс $s$ ) и ротора (индекс $r$ ), проекции которых на оси фаз $\alpha, \beta$ или $A, B, C$ дают мгновенные значения указанных величин.

Рассмотрим вначале установившийся режим работы при некоторой постоянной скорости ротора $\omega$. При этом, учитывая, что все векторы вращаются со скоростью, равной частоте сети $\omega_{1}$, они могут быть представлены комплексными величинами:

$$
\mathbf{u}_{s}=U_{m} e^{-j \varphi_{1}} e^{j \omega_{1} t}=\mathbf{U}_{s} e^{j \omega_{1} t} \text { и т.д. }
$$

В результате система уравнений (1) преобразуется к следующему виду:

$$
\begin{gathered}
\mathbf{U}_{s}=\mathbf{I}_{s} R_{s}+j \omega_{1} \Psi_{s} \quad(2 \mathrm{a}) ; \quad \Psi_{s}=L_{s} \mathbf{I}_{s}+L_{m} \mathbf{I}_{r} \\
0=\mathbf{I}_{r} R_{r}+j \omega_{1} s \Psi_{r} \quad(2 \sigma) ; \quad \Psi_{r}=L_{s} \mathbf{I}_{s}+L_{m} \mathbf{I}_{r}
\end{gathered}
$$

Здесь $s=\left(\omega_{1}-\omega\right) / \omega_{1}-$ скольжение (величина, характеризующая нагрузку).

Для дальнейшего анализа используем напряжение за активным сопротивлением

$$
\mathbf{U}_{S}^{\prime}=\mathbf{U}_{S}-\mathbf{I}_{S} R_{S}=j \omega_{1} \Psi_{S},
$$

которое определяется потокосцеплением первичной обмотки $\Psi_{S}$.

Если предположить, что $\mathbf{U}_{s}^{\prime}$ известно, а в качестве переменных использовать потокосцепления, то задача сводится к поиску единственной неизвестной - потокосцеплению $\psi_{r}$, которое определяется решением уравнения (2б), где токи $\mathbf{I}_{s}$ и $\mathbf{I}_{r}$ будут выражены через потокосцепления:

$$
\begin{aligned}
& \mathbf{I}_{s}=\Psi_{s} / L_{s}^{\prime}-\mu_{r} \Psi_{r} / L_{s}^{\prime} ; \\
& \mathbf{I}_{r}=\mu_{s} \Psi_{s} / L_{r}^{\prime}-\Psi_{r} / L_{r}^{\prime} .
\end{aligned}
$$

Здесь $L_{s}^{\prime}=L_{s} \sigma$ и $L_{r}^{\prime}=L_{r} \sigma-$ переходная индуктивность обмоток; $\sigma=1-\mu_{s} \mu_{c}-$ коэффициент рассеяния обмоток; $\mu_{s}=L_{m} / L_{s}$ и $\mu_{r}=L_{m} / L_{r}-$ коэффициенты магнитной связи обмоток статора и ротора; $\mu=\mu_{s} \mu_{r}-$ результирующий коэффициент связи.

Таким образом, уравнение (2б) преобразуется к виду

$$
0=-\mu_{s} \mu_{r} \Psi_{s}+\left(\alpha_{r}+j s \omega_{1}\right) \Psi_{r},
$$

откуда потокосцепление ротора

$$
\Psi_{r}=\frac{\mu_{s} s_{k}}{s_{k}+j s} \Psi_{s}=\frac{\mu_{s} s_{k}}{s_{k}+j s} \frac{\mathbf{U}_{s}^{\prime}}{j \omega_{1}},
$$

где $s_{k}=R_{r} / x_{r}^{\prime} ; x_{r}^{\prime}=\omega_{1} L_{r}^{\prime}$.

Далее, используя найденные потокосцепления, получим выражения для токов:

$$
\begin{gathered}
\mathbf{I}_{s}=\frac{\mathbf{U}_{s}^{\prime}}{j x_{s}^{\prime}} \frac{\sigma s_{k}+j s}{s_{k}+j s}=\frac{\mathbf{U}_{s}^{\prime}}{\mathbf{Z}_{s}} ; \\
\mathbf{I}_{r}=\frac{\mathbf{U}_{s}^{\prime}}{j x_{s}^{\prime}} \frac{-\mu_{r} s}{s_{k}+j s} .
\end{gathered}
$$

На схеме замещения (рис.1) сопротивление $\mathbf{Z}_{s}$ измеряется справа от зажимов 1-1:

$$
\mathbf{Z}_{s}=j x_{s}^{\prime} \frac{s_{k}+j s}{\sigma s_{k}+j s} \text {. }
$$

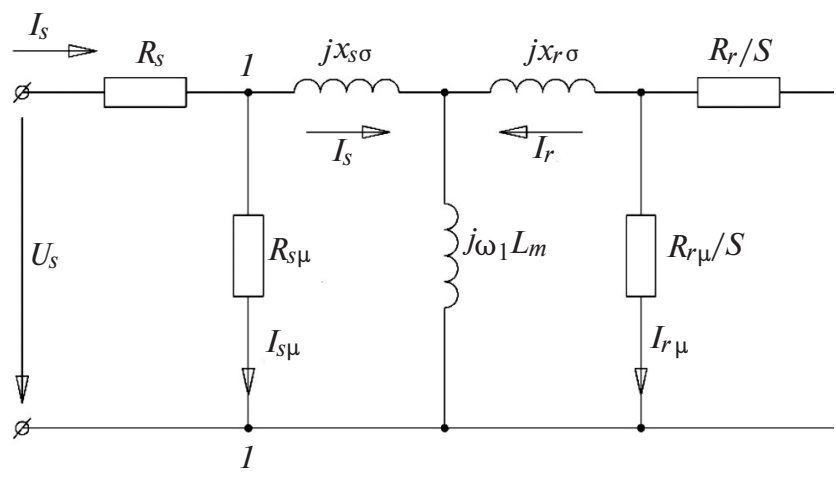

Рис. 1. Электрическая схема замещения асинхронной машины; $R_{s \mu}$ и $R_{r \mu}-$ сопротивления, обусловленные потерями в стали 


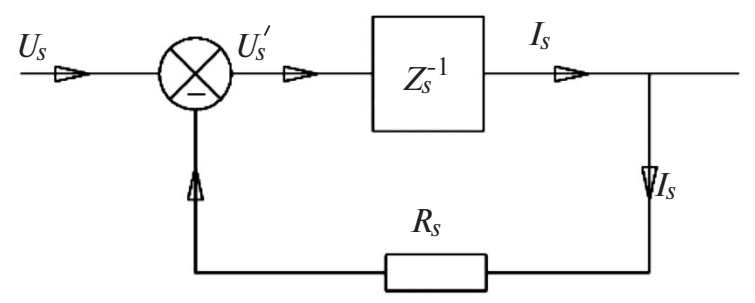

a)

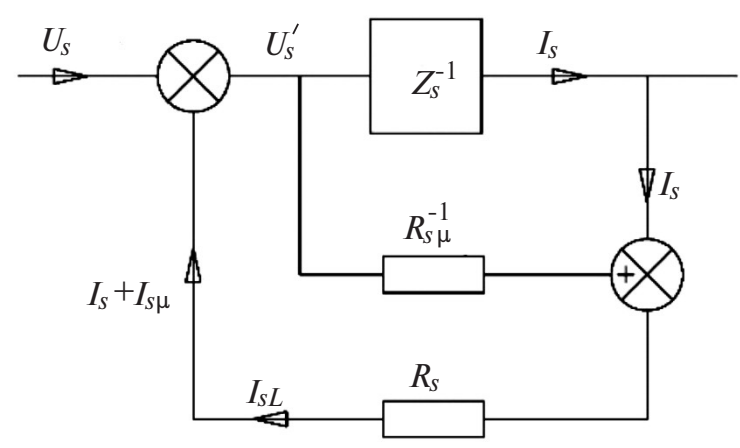

б)

Pис. 2. Структурная связь между напряжениями $\mathbf{U}_{s}$ и $\mathbf{U}_{s}^{\prime}$

До настоящего момента приведенные выводы повторяют ход решения, приведенного в [3]. Далее, для учета сопротивления авторы возвращаются к исходной системе уравнений $(2, a-2)$ и находят все величины уже в функции приложенного напряжения. Однако в этом нет необходимости, если установить непосредственную связь между напряжением сети $\mathbf{U}_{s}$ и напряжением $\mathbf{U}_{s}^{\prime}$, определенную структурной схемой рис. 2 , из которой следует, что предварительное решение можно уточнить за счет приема, использующего обратную связь.

Она широко применяется при построении автоматизированных электроприводов, но не используется в тех же целяхкак способ решения уравнений. Вместе с тем, на ее основе легко получить аналитическую зависимость между напряжениями, используя следующие соотношения:

$$
\mathbf{U}_{s}^{\prime}=\mathbf{U}_{s}-\mathbf{I}_{s} R_{s}=\mathbf{U}_{s}-\mathbf{U}_{s}^{\prime} R_{s} / \mathbf{Z}_{s},
$$

откуда находится искомая связь:

$$
\mathbf{U}_{S}^{\prime}=\frac{\mathbf{Z}_{s}}{\mathbf{Z}_{s}+R_{s}} \mathbf{U}_{s}
$$

и таким образом все ранее полученные значения токов и потокосцеплений удается представить уже как функции приложенного напряжения сети.

Для демонстрации достоверности результатов, получаемых предлагаемым методом, рассмотрим выражение (6). С учетом (9) получим

$$
\mathbf{I}_{s}=\frac{\mathbf{Z}_{s}}{\mathbf{Z}_{s}+R_{s}}=\frac{\mathbf{U}_{s}}{j x_{s}^{\prime}} \frac{\sigma s_{k}+j s}{\left(s+s s_{k s}\right)+j\left(s-\sigma s_{k} s_{k s}\right)} .
$$

Здесь $s_{k s}=R_{s} / x_{s}^{\prime}$.

Полученное выражение совпадает с решением системы уравнений (2).

То же самое можно сказать и об остальных величинах, т.е. сохраняя все преимущества, получаемые введением $\mathbf{U}_{S}^{\prime}$, за счет найденной связи (9) все искомые величины будут представлены в функции приложенного напряжения. Однако прежде попытаемся подобным образом учесть потери в стали. Они определяются основными потерями, обусловленными потоком взаимоиндукции и добавочными потерями от полей рассеяния, создаваемых токами статора и ротора. При равенстве потерь от последних потерям в стали при неподвижном роторе $(s=1)$ потери от основного потока приходились бы поровну на первичную и вторичную стороны, тогда как при синхронной скорости $(s=0)$ они должны бы снизиться примерно вдвое, но в большинстве случаев в предлагаемых схемах потери в стали от скорости ротора не зависят [4, 5]. Чтобы устранить указанный недостаток, введем сопротивления $R_{s u}$ и $R_{r \mu}$, которые должны быть включены, как показано на рис. 1. Полученная схема замещения симметрична относительно возможных источников питания, подключаемых к первичным $A-A$ и вторичным $B-B$ зажимам (например, машина двойного питания). Она отражает и тот факт, что потери в стали ротора зависят от скольжения и показывает существование вращающего момента за счет вихревых токов при разомкнутой обмотке ротора, о чем было указано в свое время в [6].

Потери в стали на первичной стороне связаны с падением напряжения на сопротивлении статора $R_{s}$. Из соотношений

$$
\mathbf{U}_{s}^{\prime}=\mathbf{U}_{s}-R_{s}\left(\mathbf{I}_{s}+\mathbf{I}_{s \mu}\right) ; \mathbf{I}_{s}=\mathbf{U}_{s}^{\prime} / \mathbf{Z}_{s} ; \mathbf{I}_{s \mu}=\mathbf{U}_{s}^{\prime} / R_{s \mu}
$$

найдем

$$
\mathbf{U}_{s}^{\prime}=\mathbf{U}_{s} \frac{\mathbf{Z}_{s}}{\mathbf{Z}_{s} a+R_{s}} ; a=1+R_{s} / R_{s \mu},
$$

где $s_{k}=R_{r}(1-b) / x_{r}^{\prime}=R_{r}^{\prime} / x_{r}^{\prime}$ необходимо определять через эквивалентное сопротивление вторичной цепи: $R_{r}^{\prime}=R_{r} /(1+b)=R_{r} /(1-b) ; b=R_{r} / R_{r \mu}$, так как $b$ много меньше единицы.

Таким образом можно записать следующие выражения:

для потокосцеплений

$$
\begin{aligned}
& \Psi_{s}=\frac{\mathbf{U}_{s}}{j \omega_{1}} \frac{s_{k}+j s}{A+j B} ; \\
& \Psi_{r}=\frac{\mathbf{U}_{s}}{j \omega_{1}} \frac{\mu_{s} s_{k}}{A+j B} ;
\end{aligned}
$$

для токов, представляя ток на зажимах машины как сумму токов, 


$$
\mathbf{I}_{s 1}=\mathbf{I}_{s \mu}+\mathbf{I}_{s},
$$

где

$$
\begin{aligned}
& \mathbf{I}_{s \mu}=\frac{\mathbf{U}_{s}}{R_{s \mu}} \frac{s_{k}+j s}{A+j B} ; \\
& \mathbf{I}_{s}=\frac{\mathbf{U}_{s}}{j x_{s}^{\prime}} \frac{\sigma s_{k}+j s}{A+j B},
\end{aligned}
$$

ток в контуре ротора

$$
\mathbf{I}_{r}=\frac{\mathbf{U}_{s}}{j x_{s}^{\prime}} \frac{-j \mu_{r} s}{A+j B}=\frac{\mathbf{U}_{s}}{j x_{r}^{\prime}} \frac{-\mu_{s} s}{A+j B} .
$$

В приведенных формулах

$$
A=s_{k} a+s s_{k s} ; B=s a-\sigma s_{k} s_{k s} .
$$

Электромагнитный момент определяется на основе известного выражения, которое для случая, когда векторы представляют комплексы, может быть записано [3]:

$$
M_{\text {эм }}=\frac{m_{1}}{2} p \operatorname{Re}\left(j \Psi_{s} \hat{\mathbf{I}}_{s}\right)=-\frac{m_{1}}{2} p \operatorname{Re}\left(j \Psi_{r} \hat{\mathbf{I}}_{r}\right) .
$$

Здесь $p$ - число пар полюсов машины; $m_{1}$ - число фаз.

Подставляя в приведенное уравнение выражения для токов и потокосцеплений, получим два выражения для момента:

$$
\begin{aligned}
& M_{\text {эм }}=\frac{m_{1}}{2} \frac{p}{\omega_{1}} \frac{U_{s}^{2}}{x_{r}^{\prime}} \mu_{s}^{2} \frac{s s_{k}}{A^{2}+B^{2}} ; \\
& M_{\text {эм }}=\frac{m_{1}}{2} \frac{p}{\omega_{1}} \frac{U_{s}^{2}}{x_{s}^{\prime}} \mu \frac{s s_{k}}{A^{2}+B^{2}},
\end{aligned}
$$

причем последнее выражение представлено через параметры первичной обмотки.

Рассмотрим предлагаемым способом переходный процесс включения асинхронной машины в сеть (бесконечной мощности), вращающейся с постоянной скоростью $\omega=$ const. Используем для этого уравнения (1), записанные в неподвижной системе координат. Проведем решение операторным методом. При нулевых начальных условиях соответствующие уравнения можно получить формальной заменой в исходной системе уравнений (1) оператора дифференцирования $d / d t$ на $p$, а действительные переменные на их изображения:

$$
\begin{aligned}
& \mathbf{u}_{S}=\mathbf{i}_{S}(p) R_{S}+p \Psi(p) ; \\
& 0=\mathbf{i}_{r}(p) R_{r}+p \Psi_{r}(p)-j \omega \Psi_{r} .
\end{aligned}
$$

Как и ранее, выразим токи $\mathbf{i}_{k}(p)$ через потокосцепления $\Psi_{k}(p)$, где $k=s, r$, и для напряжения $\mathbf{u}_{S}^{\prime}(p)=\mathbf{u}_{S}(p)-\mathbf{i}_{S}(p) R_{S}=p \Psi_{S}(p)$ задача сводится, как и ранее, к поиску $\Psi_{r}(p)$. Далее таким же образом находятся $\mathbf{i}_{s}(p), \mathbf{i}_{r}(p)$ и $\mathbf{u}_{s}^{\prime}(p)$ :

$$
\mathbf{u}_{S}^{\prime}(p)=\mathbf{u}_{S}(p) \frac{\mathbf{Z}_{S}(p)}{\mathbf{Z}_{S}(p)+R_{S}},
$$

где

$$
\mathbf{Z}_{s}(p)=p L^{\prime} \frac{p+s_{k} \omega_{1}-j \omega}{p-j \omega+s_{k} \omega_{1} \sigma} .
$$

В результате получим потокосцепления

$$
\Psi_{S}(p)=\frac{\mathbf{S}(p)}{\mathbf{N}(p)} \mathbf{U}_{s}(p) ; \Psi_{r}(p)=\frac{\mu_{S} s_{k} \omega_{1}}{\mathbf{N}(p)} \mathbf{U}_{s}(p)
$$

и токи

$$
\mathbf{i}_{S}(p)=\frac{\mathbf{S}(p)}{L_{S}^{\prime} \mathbf{N}(p)} \mathbf{U}_{S}(p) ; \mathbf{i}_{r}(p)=\frac{\mu_{S}(p-j \omega)}{L_{r}^{\prime} \mathbf{N}(p)} \mathbf{U}_{S}(p) .
$$

В полученных выражениях

$$
\begin{gathered}
\mathbf{S}(p)=p+s_{k} \omega_{1}-j \omega ; \\
\mathbf{N}(p)=p^{2}+p\left(s_{k s} \omega_{1}+s_{k} \omega_{1}-j \omega\right)+ \\
+\left(\omega_{1}^{2} s_{k} s_{k s} \sigma-j \omega_{1} \omega s_{k s}\right) .
\end{gathered}
$$

Так как приложено симметричное напряжение $\mathbf{u}_{s}=1 \mathbf{U}_{s} e^{j \omega_{1} t}$, то его изображение имеет вид

$$
\mathbf{u}_{s}(p)=\mathbf{U}_{s} \frac{p}{p-j \omega_{1}},
$$

и в соответствии с известной теоремой разложения можно найти оригиналы для всех приведенных величин (21), (22). Например, оригинал для тока статора

$$
\mathbf{i}_{S}(t)=\frac{\mathbf{U}_{S}}{L_{S}^{\prime}}\left[\frac{\mathbf{S}\left(j \omega_{1}\right)}{\mathbf{N}\left(j \omega_{1}\right)} e^{j \omega_{1} t}-\sum_{k=1}^{2} \frac{\mathbf{S}\left(p_{k}\right) e^{p_{k}}}{\left(p_{k}-j \omega_{1}\right) \mathbf{N}\left(p_{k}\right)}\right],
$$

где $p_{k}-$ корни полинома $\mathbf{N}(p)$.

Первое слагаемое (25), как легко установить с учетом (20), представляет установившееся значение тока

$$
\mathbf{i}_{S}(t)=\mathbf{I}_{S} e^{j \omega_{1} t}=\mathbf{U}_{S} e^{j \omega_{1} t}\left(\mathbf{Z}_{S}\left(j \omega_{1}\right)+R_{S}\right)^{-1},
$$

которое совпадает с ранее полученным выражением (13).

Рассмотрим далее установившийся асинхронный режим работы явнополюсной синхронной машины, вращающейся с постоянной скоростью $\omega$. Она имеет на роторе два короткозамкнутых контура: по продольной оси - успокоительный контур «уd» и контур возбуждения «f», и по поперечной оси один контур - «уq». 
Ее уравнения в осях ротора [1]:

$$
\begin{aligned}
& u_{d}=i_{d} R_{s}+p \Psi_{d}-\omega_{1}(1-s) \Psi_{q} \\
& u_{q}=i_{q} R_{s}+p \Psi_{q}-\omega_{1}(1-s) \Psi_{d}
\end{aligned}
$$

Преобразуем их, учитывая, что хотя к зажимам приложена уравновешенная система напряжений $\mathbf{u}_{s}=\mathbf{U}_{s} \mathrm{e}^{j \omega_{1} t}$, протекающие токи $i_{d}$ и $i_{q}$ из-за электрической и магнитной несимметрии будут различными:

$$
i_{d}=I_{d} \cos \left(\omega_{2} t+\alpha_{d}\right) ; i_{q}=I_{q} \sin \left(\omega_{2} t+\alpha_{q}\right),
$$

в результате в машине образуются прямо и обратно бегущие поля. Чтобы отразить указанные явления, суммируем уравнения (26), умножив последнее из них на $j$ :

$$
u_{d}+j u_{q}=R_{s}\left(i_{d}+j i_{q}\right)+(p-j \omega)\left(\Psi_{d}+j \Psi_{q}\right) .
$$

Далее, используя эйлеровские преобразования для косинусной и синусной функций и представляя мгновенные значения через соответствующие комплексы, получим:

$$
\begin{aligned}
& \mathbf{i}_{s}=i_{d}+j i_{q}=\left(\mathbf{I}_{d}+j \mathbf{I}_{q}\right) e^{j \omega_{2} t}+\left(\hat{\mathbf{I}}_{d}+j \hat{\mathbf{I}}_{q}\right) e^{-j \omega_{2} t}= \\
& =\mathbf{I}_{s 1} e^{j \omega_{2} t}+\hat{\mathbf{I}}_{s 2} e^{-j \omega_{2} t} ; \\
& \Psi_{S}=\Psi_{d}+j \Psi_{q}=\left(\Psi_{d}+j \Psi_{q}\right) e^{j \omega_{2} t}+\left(\hat{\Psi}_{d}+\right. \\
& \left.\left.+j \hat{\Psi}_{q}\right) e^{-j \omega_{2} t}=\Psi_{s 1} e^{j \omega_{2} t}+\hat{\Psi}_{s 2}\right) e^{-j \omega_{2} t} ; \\
& \mathbf{u}_{S}=u_{d}+j u_{q}=\mathbf{U}_{s 1} e^{j \omega_{2} t}+0 .
\end{aligned}
$$

Вид последнего равенства обусловлен симметрией приложенного напряжения. Подставляя последние соотношения в (27) и приравнивая члены левой и правых частей при одинаковых степенях $j \omega_{2}$ и $-j \omega_{2}$, получим уравнения:

$$
\begin{aligned}
& \mathbf{U}_{s 1}=\mathbf{U}_{s}=\mathbf{I}_{s 1} R_{s}+j \omega_{1} \Psi_{s 1} ; \\
& 0=\hat{\mathbf{I}}_{s 2} R_{s}+j \omega_{1}(2 s-1) \hat{\Psi}_{s 2},
\end{aligned}
$$

окончательный вид которых после перехода от сопряженных комплексов к комплексам:

$$
\begin{aligned}
& \mathbf{U}_{s 1}=\mathbf{U}_{s}=\mathbf{I}_{s 1} R_{s}+j \omega_{1} \Psi_{s 1} ; \\
& 0=\mathbf{I}_{s 2} R_{S}+j \omega_{1}(1-2 s) \Psi_{s 2} .
\end{aligned}
$$

Полученные уравнения показывают, что явнополюсная машина в рассматриваемом режиме отличается от обычной асинхронной машины наличием дополнительного короткозамкнутого контура, образованного обмоткой статора, замкнутой через источник питания.
Раскроем выражения потокосцеплений $\Psi_{S 1}$ и $\Psi_{s 2}$. Согласно (28) имеем:

$$
\begin{aligned}
\Psi_{d}+ & j \Psi_{q}=L_{d}\left(j \omega_{2}\right) \mathbf{I}_{d} e^{j \omega_{2} t}+L_{d}\left(-j \omega_{2}\right) \hat{\mathbf{I}}_{d} e^{-j \omega_{2} t}+ \\
& +j L_{q}\left(j \omega_{2}\right) \mathbf{I}_{q} e^{j \omega_{2} t}+j L_{q}\left(-j \omega_{2}\right) \hat{\mathbf{I}}_{q} e^{-j \omega_{2} t} .
\end{aligned}
$$

Представим операторные индуктивности в следующем виде:

$$
\begin{aligned}
& L_{d}\left(j \omega_{2}\right)=L_{0}\left(j \omega_{2}\right)+L_{2}\left(j \omega_{2}\right) ; \\
& L_{q}\left(j \omega_{2}\right)=L_{0}\left(j \omega_{2}\right)-L_{2}\left(j \omega_{2}\right),
\end{aligned}
$$

и тогда после группировки получим

$$
\Psi_{d}+j \Psi_{q}=\Psi_{s 1} e^{j \omega_{2} t}+\hat{\Psi}_{s 2} e^{-j \omega_{2} t}
$$

где

$$
\begin{aligned}
& \Psi_{s 1}=L_{0}\left(j \omega_{2}\right) \mathbf{I}_{s 1}+L_{2}\left(j \omega_{2}\right) \mathbf{I}_{s 2} ; \\
& \Psi_{s 2}=L_{2}\left(j \omega_{2}\right) \mathbf{I}_{s 1}+L_{0}\left(j \omega_{2}\right) \mathbf{I}_{s 2} .
\end{aligned}
$$

(Здесь для экономии объема вместо сопряженного комплекса для $\hat{\Psi}_{s 2}$ сразу приведен комплекс $\Psi_{s 2}$ ).

Решая последние уравнения относительно токов, получим:

$$
\begin{aligned}
& \mathbf{I}_{s 1}=\Psi_{s 1} / L_{0}^{\prime}\left(j \omega_{2}\right)-\mu_{2} \Psi_{s 2} / L_{0}^{\prime}\left(j \omega_{2}\right) ; \\
& \mathbf{I}_{s 2}=-\mu_{2} \Psi_{s 1} / L_{0}^{\prime}\left(j \omega_{2}\right)+\Psi_{s 2} / L_{0}^{\prime}\left(j \omega_{2}\right) .
\end{aligned}
$$

Здесь

$$
\begin{aligned}
& \mu_{2}=L_{2}\left(j \omega_{2}\right) / L_{0}\left(j \omega_{2}\right) ; \\
& L_{0}^{\prime}\left(j \omega_{2}\right)=L_{0}^{\prime}\left(j \omega_{2}\right)\left(1-\mu_{2}^{2}\right) ; \sigma=\left(1-\mu_{2}^{2}\right) .
\end{aligned}
$$

Подставляя выражения (30) в (29), получим после некоторых преобразований развернутые уравнения относительно токов:

$$
\begin{aligned}
& \mathbf{U}_{s 1}=\left[R_{s}+j \omega_{1} L_{q}\left(j \omega_{2}\right)\right] \mathbf{I}_{s 1}+j \omega_{1} L_{2}\left(j \omega_{2}\right) \times \\
& \times\left(\mathbf{I}_{s 1}+\mathbf{I}_{s 2}\right) ; \\
& 0=\left[R_{s} /(2 s-1)+j \omega_{1} L_{q}\left(j \omega_{2}\right)\right] \mathbf{I}_{s 2}+j \omega_{1} L_{2}\left(j \omega_{2}\right) \times \\
& \times\left(\mathbf{I}_{s 1}+\mathbf{I}_{s 2}\right),
\end{aligned}
$$

которым соответствует схема замещения, приведенная на рис. 3, где

$$
\mathbf{Z}_{q}\left(j \omega_{2}\right)=\omega_{1}\left[L_{0}\left(j \omega_{2}\right)-L_{2}\left(j \omega_{2}\right)\right] Z_{q}\left(j \omega_{2}\right) .
$$

Сравнивая эту схему с приведенной в [1], легко убедиться в их полной идентичности, несмотря на различие подходов к их получению. Это говорит о достоверности принятых исходных положений. Из нее следует, что момент от полей обратной последовательности будет создаваться только при наличии активного сопротивления $R_{s}$ и его значениепроходит через нуль при скольжении $s=0,5$. Учитывая различную роль указанного сопротивления, 


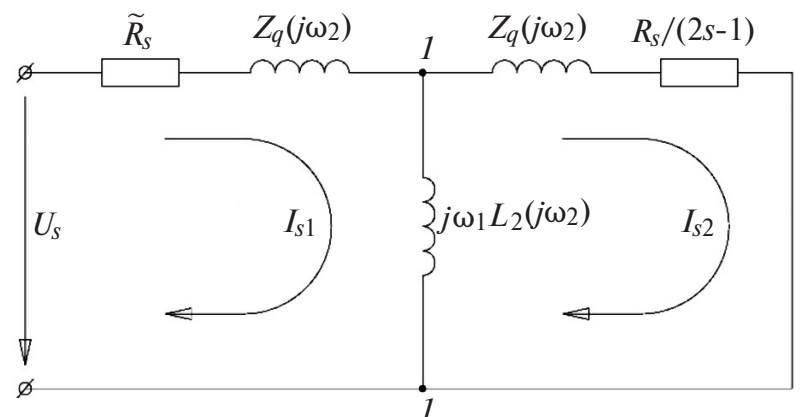

Рис. 3. Схема замещения для асинхронного режима синхронной явнополюсной машины

его принадлежность к первичной стороне будем обозначать $\widetilde{R}_{S}$ так, как показано на рис. 3 .

Уравнения (29) решаем, как и ранее, используя напряжение за активным сопротивлением и принимая в качестве независимых переменных потокосцепления, для чего используем соотношения (31):

$$
\begin{aligned}
& \mathbf{U}_{s 1}^{\prime}=\mathbf{U}_{s 1}-\mathbf{I}_{s 1} \tilde{R}_{s}=j \omega_{1} \Psi_{s 1} ; \\
& 0=-\frac{\mu_{2} R_{s}}{L_{0}^{\prime}\left(j \omega_{2}\right)} \Psi_{s 1}+\left(\frac{R_{s}}{L_{0}^{\prime}\left(j \omega_{2}\right)}+j \omega_{1} s^{\prime}\right) \Psi_{s 2},
\end{aligned}
$$

отсюда найдем

$$
\Psi_{s 2}=\frac{\mu_{2} s_{0}}{s_{0}+j s^{\prime}} \frac{\mathbf{U}_{s 1}^{\prime}}{j \omega_{1}} .
$$

Здесь $s^{\prime}=2 s-1 ; s_{0}=R_{s} / j \omega_{1} L_{0}\left(j \omega_{2}\right)$.

Далее через найденные потокосцепления определяются токи:

$$
\begin{aligned}
& \mathbf{I}_{s 1}=\frac{\mathbf{U}_{s 1}^{\prime}}{j \omega_{1} L_{0}\left(j \omega_{2}\right)} \frac{\sigma_{2} s_{0}+j s^{\prime}}{s_{0}+j s^{\prime}}=\frac{\mathbf{U}_{s 1}^{\prime}}{\mathbf{Z}_{1}} ; \\
& \mathbf{I}_{s 2}=\frac{\mathbf{U}_{s 1}^{\prime}}{j \omega_{1} L_{0}\left(j \omega_{2}\right)} \frac{-j \mu_{2} s^{\prime}}{s_{0}+j s^{\prime}} .
\end{aligned}
$$

Сопротивление $\mathbf{Z}_{1}$ измеряется справа от точек 1-1 на рис. 3, его значение

$$
\mathbf{Z}_{1}=j \omega_{1} L_{0}\left(j \omega_{2}\right) \frac{s_{0}+j s^{\prime}}{\sigma_{2} s_{0}+j s^{\prime}},
$$

используя которое, известным приемом найдем

$$
\mathbf{U}_{s 1}^{\prime}=\mathbf{U}_{s 1} \frac{\mathbf{Z}_{1}}{\mathbf{Z}_{1}+\tilde{R}_{s}},
$$

и таким образом можно представить все найденные зависимости с учетом падения напряжения на активном сопротивлении обмотки статора $\widetilde{R}_{S}$ и в итоге рассчитать электромагнитный момент в функции приложенного напряжения, если будут представлены операторные индуктивности по продольной $L_{d}\left(j \omega_{2}\right)$ и поперечной $L_{q}\left(j \omega_{2}\right)$ осям.

Найдем индуктивность $L_{q}\left(j \omega_{2}\right)$ из решения уравнений по поперечной оси. Исключая ток короткозамкнутого контура и переходя к комплексам, получим

$$
\Psi_{q}=L_{q}\left(j \omega_{2}\right) \mathbf{I}_{q}=L_{q}^{\prime} \frac{s_{\mathrm{y} q}+j s^{\prime}}{\sigma_{\mathrm{y} q} s_{\mathrm{y} q}+j s^{\prime}} \mathbf{I}_{q},
$$

где $\sigma_{\mathrm{y} q}=1-\mu_{\mathrm{y} q} ; \quad \mu_{\mathrm{y} q}=L_{a q} / L_{\mathrm{y}} L_{\mathrm{y} q} ; \quad L_{q}^{\prime}=\sigma_{\mathrm{y} q} L_{q}$; $s_{\mathrm{y} q}=R_{\mathrm{y} q} / \omega_{1} L_{q}^{\prime}$.

Поиск операторной индуктивности по продольной оси проводится аналогичным образом на основе следующих уравнений:

$$
\begin{aligned}
& \psi_{d}=L_{d} i_{d}+L_{a d} i_{\mathrm{y} d}+L_{a d} i_{f} \\
& 0=p L_{a d} i_{d}+Z_{\mathrm{y} d} i_{\mathrm{y} d}+p L_{a d} i_{f} \\
& 0=p L_{a d} i_{d}+p L_{a d} i_{\mathrm{y} d}+Z_{f} i_{f}
\end{aligned}
$$

где $Z_{\mathrm{y} d}=R_{\mathrm{y} d}+p L_{\mathrm{y} d}$ и $Z_{f}=R_{f}+p L_{f}-$ собственные сопротивления успокоительного контура по продольной оси «уd» и контура возбуждения «f».

Решая полученную систему последовательно относительно токов $i_{\mathrm{y} d}$ и $i_{f}$, найдем

$$
i_{\mathrm{y} d}+i_{f}=\frac{2 p^{2} L_{a d}^{2}-\left(\mathbf{Z}_{\mathrm{y} d}-\mathbf{Z}_{f}\right) p L_{a d}}{\mathbf{Z}_{\mathrm{y} d} \mathbf{Z}_{f}-p^{2} L_{a d}^{2}} i_{d} .
$$

Преобразуем полученный результат. Представим собственные сопротивления контуров следующим образом:

$$
\mathbf{Z}_{\mathrm{y} d}=\mathbf{Z}_{0}-\mathbf{Z}_{2} ; \mathbf{Z}_{f}=\mathbf{Z}_{0}+\mathbf{Z}_{2}
$$

и тогда для суммы токов (39) получим

$$
i_{\mathrm{y} d}+i_{f}=-\frac{2 p L_{a d}\left(\mathbf{Z}_{0}-p L_{a d}\right)}{\left(\mathbf{Z}_{0}^{2}-\mathbf{Z}_{2}^{2}\right)-p^{2} L_{a d}^{2}} i_{d} .
$$

Анализируя результат, учтем, что собственные индуктивности всех контуров по продольной оси отличаются только индуктивностями от полей рассеяния, т.е. они являются близкими значениями, тогда как значение активного сопротивления контура возбуждения $R_{f}$ много меньше аналогичного значения успокоительного контура $R_{\mathrm{y} d}$. Но при пуске обмотка возбуждения, как правило, замыкается на сопротивление, равное $(12 \div 15) R_{f}$. В результате оказывается, что $\mathbf{Z}_{0}^{2} \gg \mathbf{Z}_{2}^{2}$ и если пренебречь $\mathbf{Z}_{2}^{2}$, то вносимая ошибка по модулю и по фазе не превышает 5\%. Тогда можно записать:

$$
i_{\mathrm{yd}}+i_{f}=-\frac{2 p L_{a d} i_{d}}{\mathbf{Z}_{0}+p L_{a d}} .
$$


Возвращаясь к исходной системе уравнений по продольной оси и переходя к комплексам, найдем

$$
\Psi_{q}\left(j \omega_{2}\right)=L_{d}^{\prime} \frac{s_{\mathrm{y} d}+j s^{\prime}}{s_{\mathrm{y} d} \sigma_{\mathrm{y} d}+j s^{\prime}} \mathbf{I}_{d}=L_{d}\left(j \omega_{2}\right) \mathbf{I}_{d} .
$$

Здесь $s_{\mathrm{y} d}=R_{0} / \sigma_{\mathrm{y} d} L_{\mathrm{y} 0} ; L_{\mathrm{y} 0}=L_{a d}+0,5\left(L_{f}+L_{\mathrm{y} d}\right)$; $R_{0}=0,5\left(R_{f}+R_{\mathrm{y} d}\right) ; \quad L_{d}^{\prime}=L_{d} \sigma_{\mathrm{y} d} ; \quad \sigma_{\mathrm{y} d}=1-\mu_{\mathrm{y} d}$ $\mu_{\mathrm{y} d}=2 L_{a d}^{2} / L_{\mathrm{y} 0} L_{d}$.

Таким образом, в результате проделанных операций вид операторных индуктивностей по продольной и поперечной осям получился подобным, что значительно упрощает действия с ними. Заметим, что в нашем случае операторные проводимости $1 / j \omega_{1} L_{k}\left(j \omega_{2}\right), k=d, q$, на комплексной плоскости описывают окружности при изменении скольжения $s$ от $-\infty$ до $+\infty$. (Напомним, что $\left.\omega_{2}=\omega_{1} s.\right)$ Поэтому, чтобы использовать указанное удобство в расчетах, все ранее полученные выражения преобразованы к следующему виду:

$$
\begin{aligned}
& \Psi_{s 1}=\frac{\mathbf{U}_{s}}{j \omega_{1}}\left(R_{s} \mathbf{G}_{0}+s^{\prime}\right) \mathbf{D}^{-1} ; \\
& \Psi_{s 2}=\frac{\mathbf{U}_{s}}{j \omega_{1}} R_{s} \mathbf{G}_{2} \mathbf{D}^{-1} ; \\
& \mathbf{I}_{s 1}=\mathbf{U}_{s} \mathbf{G}_{0}\left(R_{s} \mathbf{Y}_{0}+s^{\prime}\right) \mathbf{D}^{-1} ; \\
& \mathbf{I}_{s 2}=-\mathbf{U}_{s} \mathbf{G}_{2} s^{\prime} \mathbf{D}^{-1} .
\end{aligned}
$$

Здесь $\mathbf{D}=\left(R_{s} \mathbf{G}_{0}+s^{\prime}\right) a+\widetilde{R}_{s} \mathbf{G}_{0}\left(R_{s} \mathbf{Y}_{0}+s^{\prime}\right)$;

$$
\begin{aligned}
& \mathbf{G}_{0}=\frac{1}{2 j}\left[\frac{1}{X_{q}\left(j \omega_{2}\right)}+\frac{1}{X_{d}\left(j \omega_{2}\right)}\right] ; \\
& \mathbf{G}_{2}=\frac{1}{2 j}\left[\frac{1}{X_{q}\left(j \omega_{2}\right)}+\frac{1}{X_{d}\left(j \omega_{2}\right)}\right] ; \\
& \mathbf{Y}_{0}=\frac{2}{j\left[X_{q}\left(j \omega_{2}\right)+X_{d}\left(j \omega_{2}\right)\right]} .
\end{aligned}
$$

Электромагнитный момент определяется на основе известного выражения, которое для случая, когда векторы представляют комплексы, имеет известный вид $M_{\text {эм }}=0,5 m_{1} p \operatorname{Re}\left(j \Psi_{S} \hat{\mathbf{I}}_{s}\right)$, раскрывая в котором комплексы

$$
\begin{aligned}
& \Psi_{S}=\Psi_{s 1} e^{j \omega_{2} t}+\Psi_{s 2} e^{-j \omega_{2} t} ; \\
& \hat{\mathbf{I}}_{S}=\hat{\mathbf{I}}_{s 1} e^{-j \omega_{2} t}+\hat{\mathbf{I}}_{s 2} e^{j \omega_{2} t},
\end{aligned}
$$

получим

$$
M_{\text {эм }}=M_{\text {эм } 1}+M_{\text {эм } 2}+M_{\text {эм } 3}+M_{\text {эм } 4}
$$

где сумма первых двух слагаемых представляет асинхронные моменты, обусловленные векторами, вращающимися с одинаковыми скоростями при любом скольжении:

$$
M_{\mathrm{a}}=0,5 m_{1} p \operatorname{Re} j\left(\Psi_{s 1} \hat{\mathbf{I}}_{s 1}+\hat{\Psi}_{s 2} \mathbf{I}_{s 2}\right) .
$$

Среднее значение двух последних слагаемых не равно нулю только при синхронной скорости

$$
M_{\mathrm{c}}=0,5 m_{1} p \operatorname{Re} j\left(\Psi_{s 1} \mathbf{I}_{s 2}+\hat{\Psi}_{s 2} \hat{\mathbf{I}}_{s 1}\right) \text {. }
$$

Здесь первое слагаемое представляет так называемый реактивный момент, он обусловлен различной магнитной проводимостью по осям ротора, приводящей к появлению зубцовой гармоники, число полюсов которой соответствует основной волне МДС статора. Она же является причиной и второй составляющей синхронного момента, действующего встречно первой.

Вывод. Рассмотренные примеры показывают, что применение принципа обратной связи для учета активного сопротивления первичной обмотки дает возможность ввести в качестве независимых переменных вместо токов потокосцепления контуров и снизить порядок исследуемой системы уравнений на единицу. При этом при поиске решения сохраняются известные преимущества, связанные с пренебрежением активного сопротивления первичной обмотки, а с помощью приема, основанного на принципе обратной связи, обеспечивается строгий учет влияния последнего. Подобный подход может быть использован и при анализе механических систем, поскольку в последних аналогом потокосцеплению является импульс, а току - скорость [4].

\section{СПИСОК ЛИТЕРАТУРЫ}

1. Уайт Д., Вудсон Г. Электромеханическое преобразование энергии. - М.: Энергия, 1964, 526 с.

2. Казовский Е.Я. Переходные процессы в электрических машинах переменного тока. - Изд. АН СССР, 1962, 624 с.

3. Ковач К., Рац И. Переходные процессы в машинах переменного тока. - М.: ГЭИ, 1963, 738 с.

4. Жуловян В.В. Электрические машины: электромеханическое преобразование энергии. - М.: Юрайт, 2017, 427 с.

5. Гурин Я.С., Кузнецов Б.И. Проектирование серий электрических машин. - М.: Энергия, 1978, 480 с.

6. Рихтер Р. Электрические машины. Т. 4. Индукционные машины. - М.; Л.: ГЭИ,1939, 472 с.

[13.09.2017]

А в $m$ о р: Жуловян Владимир Владимирович закончил Томский политехнический институт в 1954 г. по специальности «Электрические машины и аппараты». В 1978 г. защитил докторскую диссертацию «Высокомоментные двигатели переменного тока с электромагнитной редукцией частоты вращения» в Московском энергетическом институте. Профессор кафедры электротехнических комплексов Новосибирского государственного технического университета. 
Elektrichestvo (Electricity), 2017, No. 12, pp. 29-36

DOI:10.24160/0013-5380-2017-12-29-36

\section{Applyingthe Feedback Principle for Solving the Equations of Transformers and Electrical Machines}

ZHULOVYAN Vladimir V. (Novosibirsk State Technical University) - Professor, Dr. Sci. (Eng.)

Anapproach is suggested according to which the primary winding resistance istakeninto account using the well-known feed back principle. The search procedure involves two stages. In the first stage, a voltage source is applied behind the resistance. Then, if flux linkages of the loops are taken as independent variables instead of currents, the order of the equation system to be solved is decreased by unity, a circumstance due to which both the search and the form of sought solutions become essentially simpler. Atthesecondstage, a mathematically rigorous correlation between the conditional and actual voltage across the terminals is found using atechnique known as the feed back principle, as a result of which the preliminarily found quantities are presented as functions of the actual voltage across the terminals. Examples demonstrating the validity and effectiveness of the proposed approach are given as applied to a number of problems concerned with studying steady-state and transient modes of electrical machines and transformers.

$\mathrm{K}$ e y w o r d s : electrical machines, primary winding, resistance, feedback, equation system's order

REFERENCES

1. White D., Woodson G. Electromechanical energy conversion. Moscow, Publ. «Energiya», 1964, 526 p.

2. Kazovsky E.Y. Transient processes in electrical machines of alternating current. Publ. USSR Academy of Sciences, 1962, 624 p.

3. Kovács K., Rácz I. Transients in machines AC. Moscow, Gosenergoizdat,1963, 738 p.
4. Zhulovyan V. Electrical machines: Electromechanical energy conversion. - Moscow, Publ. «Yurayt», 2017,427 p.

5. Gurin .J.S., Kuznetsov B.I. Series of electric machines designing. Moscow, Publ. «Energiya», 1978, 480 p.

6. Richter R. The electric machine. Vol. 4. The induction machine. Moscow; Leningrad, Gosenergoizdat, 1939, 472 p.

[13.09.2017] 